\title{
Insurance Based Lie Detection: Enhancing the Verifiability Approach with a Model Statement Component
}

\author{
Adam C Harvey* \\ Aldert Vrij \\ Sharon Leal \\ Marcus Lafferty \\ Department of Psychology, University of Portsmouth, UK \\ Galit Nahari \\ Department of Criminology. Bar-Ilan University, Israel
}

Key Words: Insurance interviewing; lie detection; eliciting cues

*Address for correspondence: Adam Charles Harvey, Department of Psychology, University of Portsmouth, King Henry Building, King Henry I Street, Portsmouth, PO1 2DY. UK. adam.harvey@port.ac.uk. Telephone: +44 239284661 


\section{Insurance Based Lie Detection: Enhancing the Verifiability Approach with a Model Statement Component}

Individuals lie for many reasons, including for self-orientated financial gain (DePaulo et al., 1996; Ekman, 1985; Vrij, 2008). Insurance fraud is deemed acceptable to many policyholders, with $20 \%$ of surveyed individuals admitting they would consider submitting an exaggerated or fabricated insurance claim in the future (Association of British Insurers [ABI], 2009). In response, the UK insurance industry invests considerable resources - over $£ 200$ million per year - in an attempt to combat fraud. Nevertheless, undetected insurance fraud in the UK is estimated to be in excess of $£ 2.1$ billion per year (ABI, 2009). It is therefore unfortunate that little research is conducted regarding lie detection within financial settings, with the majority of deception literature narrowly focusing upon police-suspect interviewing (Vrij \& Granhag, 2012; 2014; Vrij, Granhag \& Porter, 2010).

Evidence suggests that the core theoretical conclusion derived from the wider deception literature (e.g., Vrij \& Granhag, 2012; Vrij \& Granhag 2014, Vrij, Fisher \& Blank, 2015) may also apply to insurance claims settings. That is, that the weak objective cue hypothesis (Hartwig \& Bond, 2011), which implies that without active elicitation an insufficient number of reliable credibility cues is available to facilitate accurate deception detection (DePaulo et al., 2003; Levine, 2014, Vrij \& Granhag, 2012), may also apply to insurance claims settings (Harvey, Vrij, Nahari \& Ludwig, 2016; Leal, Vrij, Warmelink, Vernham, \& Fisher, 2015; Nahari \& Vrij, 2014). Therefore, what appears to be required to improve lie detection accuracy is employing proactive interview protocols that impose manipulations to elicit and magnify cues to deception (Harvey et al., 2016; Leal et al., 2015; Vrij, Nahari, Isitt, \& Leal, 2016). The purpose of the current study is to extend the embryonic research on verbal lie detection within the insurance claim domain by exploring a potential refinement of the 
Verifiability Approach (VA), a verbal veracity tool introduced by Nahari, Vrij and Fisher (2014a, 2014b). Harvey et al. (2016) demonstrated that providing interviewees information about the VA's function is necessary to facilitate lie detection in the insurance claims setting using the VA. Extending that research, the current study explores a novel protocol to further enhance the VA's usefulness in insurance claims settings - model statement. Specifically, we tested the hypothesis that supplementing the VA with a 'tailored' model statement (a detailed example statement comprised of checkable information) to actively prime claimants to provide statements with many verifiable details will facilitate superior lie detection with the VA.

\section{The Verifiability Approach}

The VA is a verbal veracity tool that examines the frequency of checkable details disclosed in suspect's statements during interviews (Nahari et al., 2014a, 2014b). According to the VA, liars and truth tellers exercise different verbal strategies in respect to checkable information (Harvey et al., 2016; Vrij et al., 2016). Truth tellers tend to be verbally forthcoming, freely disclosing checkable details they can remember (Nahari et al., 2014a; see also Hartwig, Granhag, \& Strömwall, 2007; Hartwig, Granhag, Strömwall \& Doering, 2010). In contrast, according to the VA, in interview settings liars are confronted by an information management dilemma and their verbal behaviour is constrained by two conflicting motivations (Nahari et al., 2014a, 2014b). First, liars are motivated to convey an honest impression. To maximise their chance of being judged as credible they wish to provide statements rich in detail (Hartwig et al., 2007; Nahari, Vrij et al., 2012; Masip \& Herrero, 2013; Strömwall et al., 2006), as statements richer in detail are more likely to be judged as credible (Bell \& Loftus, 1989; Johnson, 2006; Johnson, Foley, Suengas, \& Raye, 1988). Second, liars are motivated to minimise the investigator's opportunities to check and potentially falsify their statement, exposing their deception (Masip \& Herrero, 2013; Nahari 
et al., 2012, 2014a). The latter makes sense as investigators frequently check the veracity of what is disclosed by comparing statements to evidence (e.g., Hartwig, Granhag, Strömwall \& Vrij, 2005). According to the VA, a potential solution to the information management dilemma is for liars to strategically withhold disclosing checkable detail. This assumption has received considerable empirical support and both the frequency and relative percentage of verifiable details have emerged as a promising verbal cue to deception (Harvey et al., 2016; Nahari et al., 2014a, 2014b, Nahari, Leal et al., 2014; Vrij et al., 2016).

The 'Information Protocol' (IP; Nahari et al., 2014b; Harvey et al., 2016) is a component of the full VA procedure (Harvey et al., 2016; Vrij, Vrij, Nahari et al., 2016; Nahari \& Vrij, 2014) and informs interviewee's of the importance of including checkable information in their statements (see Table 1 for a detailed breakdown of the individual IP components).

\section{[Insert Table 1 about here]}

Originally, the IP was developed to examine the robustness of the VA to countermeasures, i.e., whether informing participants about the VA's functionality impairs the efficiency of the test (Nahari et al., 2014b). Research indicates that the VA is not simply resistant to countermeasures, informing interviewees about how the VA works actually increases its accuracy (Nahari et al., 2014b). That is, the number of verifiable details reported by liars does not vary as a function of the IP provision, but does vary among truth tellers, with truth tellers providing more verifiable details when an IP is provided compared to when no IP is provided (Nahari et al., 2014b; Harvey et al., 2016). In the insurance claims setting, the provision of the IP appears critical to the ability of the VA to accurately distinguish between fabricated and genuine statements (Harvey et al., 2016). When no IP is utilised the VA has been unable to accurately classify liars and truth tellers, but when the IP is utilised the VA can successfully discriminate between liars and truth tellers (Harvey et al., 2016; 
Nahari et al., 2014b; Vrij et al., 2016).

\section{Encouraging Suspects to Say More}

Verbal differences between liars and truth tellers are magnified if interviewees are encouraged to provide longer statements (Leal et al., 2015; Mann et al., 2013; Shaw et al., 2015; Vrij et al., 2007, Vrij et al., 2012, Vrij \& Granhag, 2014). Truth tellers do not initially say all they know (Vrij, Hope, \& Fisher, 2014) and there is room for them to provide more information if encouraged to do so. Critically, liars may not be able to give as much detail as truth tellers, because they lack the imagination to provide a wealth of detail that also sounds plausible or they may reluctant to do so because they fear that these additional details may provide leads to investigators that can expose their deception (Vrij, 2015). The latter point particularly applies to verifiable detail, because truth tellers can be forthcoming with verifiable detail, whereas liars must be avoidant with verifiable detail (Nahari et al., 2014a, 2014b; Vrij et al., 2016). As such, eliciting longer statements from suspects should plausibly enhance the difference between liars and truth tellers in terms of verifiable detail.

One potential means of eliciting additional information from suspects within the VA framework is via a model statement prime (Leal et al., 2015). A model statement is a detailed example of an unrelated topic to that of the interview, designed to modify the interviewee's expectations of what is required from their statement (Leal et al., 2015). Social comparison theory provides a theoretical explanation for the working of a model statement in interview settings (Festinger, 1954, see also Cialdini's social proof), and states that in the absence of objective information, people will compare themselves to others. This means that in an interview setting where objective information about how much detail is required is not available, interviewees will use the model statement as a point of reference, and if the model statement is detailed, they will become detailed themselves. In alignment with this, research has shown that providing interviewees with model statements containing many details made 
interviewees to calibrate their verbal output and to incorporate many details into their own statements (Bogaard, Meijer \& Vrij, 2014, Leal et al., 2015). Model statements can, in theory, be constructed to incorporate specific types of detail. As such, a model statement that contains many verifiable details may result in a divergence of verbal behaviour between truth tellers and liars with respect to verifiable detail.

Theoretically, truth teller's verbal behaviour is a function of the 'phenomenology of innocence' (Jordan \& Hartwig, 2013). Truth tellers believe their innocence is self-evident, known as the illusion of transparency (Gilovich, Savitsky, \& Medvec, 1998), and they thus freely disclose information, adopting a 'tell it as it is' verbal strategy (Hartwig, Granhag \& Strömwall 2007; Hartwig et al., 2010; Strömwall et al., 2006; Vrij, Mann, Leal, \& Granhag, 2010). According to the VA, truth tellers can freely disclose any verifiable detail they can recall (Nahari et al., 2014a, 2014b). Supplementing the full VA procedure, including the information protocol, with a model statement that contains many verifiable details (henceforth; verifiable model statement) may elicit additional checkable details (compared to the full VA) from truth tellers for two reasons. First, a model statement may motivate interviewee's to disclose more detail generally, and truth tellers to disclose more verifiable details specifically. Second, a model statement containing multiple examples of verifiable details may provide both liars and truth tellers with a more elaborate and concrete understanding of what information is and is not verifiable. According to the former 'motivation hypothesis', whereas the VA's IP manipulation primarily allows truth tellers to better calibrate the type of information they should disclose (Harvey et al., 2016; Nahari et al., 2014b), the verifiable model statement should allow truth tellers to also better calibrate the quantity of detail that should disclose (Bogaard et al., 2014; Leal et al., 2015). That is to say, the model statement motivates interviewee's generally to calibrate their verbal output to match the model statement's level of detail, and thus to disclose longer, more detailed 
statements (Bogaard et al., 2014; Leal et al., 2015). Critically, when motivated to provide longer and more detail statements, truth tellers can disclose any verifiable detail they can remember, whereas liars, according to the information management dilemma, cannot (Nahari et al., 2014a, 2014b, Nahari \& Vrij, 2014; Vrij et al., 2016). The solution for liars is to disclose additional uncheckable information. According to the second, 'calibration hypothesis' providing a verifiable model statement may provide suspects a more concrete and elaborate understanding of what information is, in principle, verifiable. This superior ability of the interviewee to understand the difference between checkable and uncheckable detail from the perspective of the interviewer then translates to a superior ability of truth tellers and liars to execute (i.e., calibrate) their verbal output relative to their respective forthcoming and evasive strategies.

According to both rationales, liars face a problem when confronted with a model statement that contains many verifiable details, as providing verifiable details is something they cannot or do not want to do. Liars may ignore the model statement prime. This is likely to be an ineffective counter strategy as liars are likely to realise, as a consequence of the model statement, that they are expected to provide more detail to be judged as truthful (Leal et al., 2015). A more likely solution for liars is to ignore the motivation to disclose verifiable details but to disclose additional uncheckable information instead. Naturally, they then do not follow the model statement in terms of the quality of detail they are supposed to provide (verifiable detail) but at least they follow the model statement in terms of the quantity of detail they are supposed to provide.

The study used a 2 (Veracity: Truth/ Lie) X 2 (Interview Type: VA with MS/ VA no MS) between-subjects design. It was hypothesised that truth tellers will report more verifiable detail than liars, particularly in the Verifiability Approach (VA) with model statement (MS) condition (Hypothesis 1) and that liars will report more unverifiable details than truth tellers, 
particularly in the VA with MS condition (Hypothesis 2). Furthermore, on the basis of the above predictions, we expected that the percentage of verifiable detail (verifiable detail / total detail [verifiable plus unverifiable]) to be more diagnostic of veracity in the VA with MS condition than in the VA without MS condition (Hypothesis 3).

\section{Method}

\section{Participants}

A total of 80 volunteers, comprising of 36 females and 44 males, aged between 18 and 39 years $(M=20.07$ years, $S D=2.35)$, from the University's undergraduate, postgraduate and staff communities, participated in the study. A total of 27 participants reported accidental damage ( 21 truth tellers), 29 reported theft (10 truth tellers) and the remaining 24 reported loss (9 truth tellers).

\section{Procedure}

Participants were recruited via adverts on the University's online participant pool. We solicited individuals for a study on lie detection in an insurance claims setting. Individuals arrived at the laboratory at pre-arranged times and were informed that the study was about detecting deception within insurance claim settings. Each participant was given an information sheet about the study and was asked to sign an informed consent form.

To allocate participants to the truthful or deceptive condition, each participant was asked the following: 'Has any item of yours, worth between $£ 100$ and $£ 1000$, been lost, damaged or stolen in the last three years?' Participants who answered 'yes' were allocated to the truthful condition, while participants who answered 'no' were allocated to the lying condition. This method was also used in previous research (Harvey et al., 2016; Leal et al., 2015; Vrij et al., 2016). Data collection continued until 40 participants who had genuinely experienced a loss (truth tellers) and 40 participants who had not experienced a loss (liars) had been recruited with additional participants discarded during the recruitment stage once 
the conditions where full. No difference in age $t(78)=1.24, p=0.219$, or gender, $\chi^{2}(1, \mathrm{n}=80)$ $=0.000$, phi $=0.000, p=1.000$, emerged between truth tellers and liars.

Truth tellers $(n=40)$ were asked to imagine that they were submitting a claim about their genuine lost/stolen item to an insurance company. Liars $(n=40)$ were asked to imagine that they were submitting a claim about their fabricated lost, damaged or stolen item to an insurance company. All the participants, truth-tellers and liars, were informed that the interview itself would be carried out by an automated computer program and that they should follow the on screen instructions. We constructed the interview using Qualtrics (www.qualtrics.com), an online survey authorship and software provider. The experimenter informed all the participants (liars and truth tellers) that their objective was to convince the human analyst (who would read their statement) that they were being honest and the reported loss was real.

The participants were randomly allocated to the VA without MS condition (i.e., with the instruction used in previous research; Nahari et al., 2014b) or the VA with MS condition. The participants in the VA without MS condition ( $n=20$ liars, $n=20$ truth tellers) were asked to start the interview and follow the on-screen instructions. The instructions began by asking participants (henceforth, claimants) to read the guidelines carefully because once they progressed they would be unable to return to a previous page. Then, the instructions informed claimants that the analyst would read their statement carefully and would check if the details provided could be verified:

"We know from research that liars prefer to avoid providing details that can be verified whereas truth tellers prefer to provide details that can be verified. Therefore the analyst will check carefully to what extent the details you provide can be verified".

Additionally, the program explained that verifiable details include: i) activities with 
identifiable or named persons who the interviewer can consult, ii) activities that have been witnessed by identifiable or named persons who the interviewer can consult, iii) activities that the interviewee believes may have been captured on CCTV and iv) activities that may have been recorded or documented, such as using debit cards, mobile phones, or computers (Nahari et al., 2014a, 2014b). Details that do not meet at least one of these criteria are classified as unverifiable (Nahari, Leal, Vrij, Warmelink, \& Vernham, 2014). Claimants were also informed that the analyst may check the occurrence of some or all of the details provided in the statements after the interview.

Claimants in the VA with MS condition ( $n=20$ liars, $n=20$ truth tellers $)$ were provided an additional instruction after the information given to those in the VA without MS condition. It was stated that it can be difficult for claimants to gauge the appropriate level of detail to report and what is classified as checkable detail. Therefore, they should read carefully a 'model statement'; a detailed short statement providing an indication of the appropriate level of detail to provide that contains examples of checkable information. These instructions were adapted from previous research (Leal et al., 2015). The model statement was 377 words in length. A brief extract from of the model statement is included below. For clarity we indicate ( $\underline{\text { underlined) }}$ ) what the verifiable details were but this information was not provided to the participants:

'...I got Charlotte to take a photo of me with Tom's formula two car and then got one of the pit girls to take a photo of Charlotte and me with our VIP passes and then sent both pictures

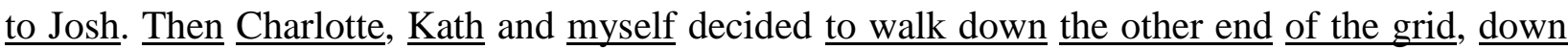
to position 22. It's a fair distance from pole position to last position so as we walked down $\underline{\text { I }}$ made another video on my phone...'

After the model statement information, the VA with MS interviewing condition were identical to the VA without MS condition. Claimants in both conditions were asked to type, 
in as much detail as possible, their statement. Once claimants indicated they were finished (i.e., ready to submit their claim), the program instructed them to inform to the experimenter they had completed the interview.

After typing their statements, the participants completed a post-interview questionnaire. The questions included the following manipulation check: 'To what extent is your account based on a truthful experience?' Participants were asked to respond on an 11-point Likert scale, ranging from $0 \%$ to $100 \%$ of their statement being based upon a truthful experience. Participants were also asked 'To what extent were you motivated to appear convincing in your statement' and to rate their motivation on a 7-point Likert scale ranging from 1 (not at all motivated) to 7 (extremely motivated).

\section{Coding the verifiability of statements and inter-rater reliability.}

Both the main and second (inter-rater) coder have coded extensively within the department previously. Training was originally overseen by the Second and Last authors of this study over a 3-week period, and consisted of text examples and definitions of the criteria, together with coding tasks to complete. The coding protocol adopted was identical to Harvey et al. (2016). All statements were first rated by one coder (blind to the experimental conditions) who scored the frequency occurrence (rather than a scale response rating; see Nahari, 2016) of overall detail. For clarity, we provide examples of each detail type below, with each unit of analysis underlined. Overall detail was defined using Reality Monitoring criteria definitions (e.g. Vrij 2008, 2015) as the sum total of perceptual detail (information about what was seen, heard, felt and smelt during the described activities; e.g. 'The security guard shouted and pointed), spatial detail (information about locations or the arrangement of persons and/or objects; e.g. 'I was to the left of Oscar') and temporal details (information about when the event happened and explicit descriptions of the sequence of various events; e.g. 'I then called my mother and after that my insurer') that potentially could or could not be 
verified. This resulted in 'information units' that could be a single word or combinations of words, depending upon the meaning. Verifiable details were: activities the interviewee said were (i) carried out with (an)other identifiable person(s) who can be traced (e.g. staff at a named establishment: 'I told the Manager at the Library' or a named person: 'I danced with Josh Jennings'), (ii) witnessed by at least one other identifiable and traceable person (e.g. 'the manager was present' or 'Josh Jennings was with me'), (iii) recorded by CCTV cameras, or (iv) documented and therefore checkable (e.g., phone calls, cash withdrawal from ATM machines; I phoned the police') (Nahari et al., 2014a, 2014b). Details that do not meet at least one of these criteria are classified as unverifiable (Nahari, Leal et al., 2014). Importantly, the definitions for verifiable and unverifiable details that were used for coding were identical to those given to the participants in the IP instructions.

A second coder (also blind to the veracity of the statements) coded a random selection of 16 statements (20\%). Inter-rater reliabilities between the two coders for the occurrence frequency of detail that could or could not be verified were measured via intra-class correlation coefficients (ICC). The ICC was high and therefore satisfactory for verifiable detail $[\mathrm{ICC}]=.98$, unverifiable detail $[\mathrm{ICC}]=.88$ and total detail (the sum of verifiable and unverifiable detail) $[\mathrm{ICC}]=.95$.

Additionally, we calculated the ratio of verifiable detail (total verifiable detail/total [verifiable plus unverifiable] detail) per statement. The ratio of verifiable detail per statement could range from 0 to 1 with a score of .50 indicating the same number of verifiable and unverifiable details in a statement. A score above .50 indicates the participant reported more verifiable than unverifiable details, whereas a score below .50 indicates the participant reported less verifiable details than unverifiable details.

\section{Results}

\section{Manipulation Checks}




\section{Veracity manipulation check.}

Truthful claimants reported their overall truthfulness rating as high $(M=95.75 \%, S D=$ 9.84\%, $95 \%$ CI [92.60\%, 98.90\%]). Deceptive claimants reported a significantly lower truthfulness rating $(M=29.75 \%, S D=31.01 \%, 95 \%$ CI $[19.83 \%, 39.63 \%]), t(46.779)=$ $12.831, p<0.001, d=2.87$. This supports the validity of the veracity manipulation.

\section{Motivation.}

The reported motivation of the participants was high $(M=5.59, S D=1.18,95 \% \mathrm{CI}$ $[5.33,5.84])$. A 2 (Veracity) X 2 (Information Protocol Type) analysis of variance (ANOVA) showed no main effect for Veracity or Information Protocol Type, and no Veracity X Information Protocol Type interaction, all $F$ 's $<.179$, all $p$ 's $>.673$.

\section{Number of words.}

On average the claimants wrote 202.06 words $(S D=120.83,95 \%$ CI $[177.93,227.99])$. A 2 (Veracity) X 2 (Information Protocol Type) analysis of variance (ANOVA) showed no main effect for Veracity, $F(1,76)=1.50, \mathrm{MSE}=18007.54, p=0.224, \eta_{\mathrm{p}}{ }^{2}=.052$. A main effect emerged for Information Protocol Type, $F(1,76)=19.19$, MSE $=230020.74, p<$ $0.001, \eta_{p^{2}}=.20$. Claimants exposed to the VA with MS $(M=255.69, S D=135.95,95 \%$ CI [112.25, 297.14]) provided longer statements than claimants exposed to the VA without MS $(M=149.12, S D=73.09,95 \%$ CI $[126.77,171.99])$. The Veracity X Information Protocol Type interaction was not significant, $F(1,76)=0.01, \mathrm{MSE}=112.50, p=0.923, \eta_{\mathrm{p}}{ }^{2}=.00$. Whilst both liars and truth tellers were equally affected by the inclusion of a model statement, this was not due to their veracity status. Therefore, further analyses do not need to include word count as a covariant.

\section{Hypothesis Testing}

\section{Number of verifiable details.}

A 2 (Veracity) x 2 (Information Protocol Type) analysis of variance (ANOVA) with the 
number of verifiable details as the dependent variable revealed a significant main effect for Veracity, $F(1,76)=15.36, \mathrm{MSE}=11463.62, p<0.001, \eta_{\mathrm{p}}^{2}=.17$, showing that truth tellers $(M=37.35, S D=37.18,95 \%$ CI $[27.23,49.30])$ reported more verifiable details than liars $(M$ $=12.78, S D=16.35,95 \%$ CI $[7.74,18.06])$. There was also a statistically significant main effect for Information Protocol Type, $F(1,76)=3.90, \mathrm{MSE}=2909.52, p=0.026, \eta_{\mathrm{p}}^{2}=.05$, showing that claimants exposed to the VA with MS $(M=31.70, S D=38.12,95 \%$ CI [21.23, 43.55]) reported more verifiable details than claimants exposed to the VA without MS ( $M=$ $18.43, S D=20.39,95 \%$ CI $[12.44,24.73])$. The Veracity x Information Protocol Type interaction effect was also statistically significant, $F(1,76)=6.30$, $\mathrm{MSE}=4705.05, p=$ $0.014, \eta_{\mathrm{p}^{2}}=.08$. In the VA with MS condition, truth tellers $(M=50.38, S D=42.80,95 \% \mathrm{CI}$ $[33.23,70.64])$ reported more verifiable details than liars $(M=11.05, S D=16.01,95 \% \mathrm{CI}$ $[4.20,18.37]), t(25.975)=3.919, p=0.001, d=1.22$. In contrast, there was no significant difference between truth tellers $(M=22.95, S D=23.30,95 \%[13.79,32.99])$ and liars who were exposed to the VA without MS $(M=14.33, S D=16.88,95 \%$ CI $[8.21,22.14])$, $t(32.555)=1.327, p=0.097, d=0.42$. This supports Hypothesis 1 .

\section{Number of unverifiable details.}

A 2 (Veracity) x 2 (Information Protocol Type) analysis of variance (ANOVA) with unverifiable details as the dependent variable revealed a significant main effect for Veracity, $F(1,76)=79.83, \mathrm{MSE}=49324.03, p<0.00, \eta_{\mathrm{p}}{ }^{2}=.51$, showing that liars $(M=77.70, S D=$ $39.45,95 \%$ CI $[66.34,89.97])$ reported more unverifiable details than truth tellers $(M=$ 29.33, $S D=14.79,95 \%$ CI [24.85, 34.37]). The analysis revealed a significant main effect for Information Protocol Type, $F(1,76)=23.47, \mathrm{MSE}=14501.73, p<0.001, \eta_{\mathrm{p}^{2}}=.24$, showing that claimants in the VA with MS condition $(M=65.75, S D=46.16,95 \%$ CI [51.64, 80.62]) reported more uncheckable details than claimants in the VA without MS condition ( $M$ $=41.28, S D=23.18,95 \% \mathrm{CI}[34.14,48.71])$. Furthermore, the Veracity x Information 
Protocol Type interaction was significant $F(1,76)=12.56, \mathrm{MSE}=7762.50, p=0.001, \eta_{\mathrm{p}}{ }^{2}=$ .14. In the VA with MS condition, liars $(M=102.21, S D=40.65,95 \%$ CI $[83.92,120.55])$ reported more unverifiable detail than truth tellers $(M=32.76, S D=16.16,95 \%$ CI $[26.19$, $40.13]), t(23.093)=-6.965, p<0.001, d=2.25$. Also in the VA without MS condition, liars $(M=55.52, S D=21.45,95 \% \mathrm{CI}[47.00,65.00])$ reported more unverifiable detail than truth tellers $(M=25.53, S D=12.44,95 \%$ CI $[20.07,31.109), t(32.631)=-5.472, p<0.001, d=$ 1.71. The effect was the most pronounced in the VA with MS condition, which supports Hypothesis 2.

\section{Percentage of Verifiable details}

A 2 (Veracity) x 2 (Information Protocol Type) analysis of variance (ANOVA) with percentage of verifiable details as the dependent variable revealed a significant main effect for Veracity, $F(1,76)=38.718, \mathrm{MSE}=1.957, p<0.001, \eta_{\mathrm{p}^{2}}=.34$, showing that liars $(M=$ $.13, S D=0.16,95 \%$ CI $[.06, .20])$ reported a lower percentage of verifiable detail than truth tellers $(M=.45, S D=.29,95 \%$ CI $[.37, .51])$. No main effect for Information Protocol Type emerged, $F(1,76)=.274$, MSE $=.014, p=0.602, \eta_{p^{2}}=.01$, with claimants in the VA with MS condition $(M=.31, S D=.30,95 \% \mathrm{CI}[.23, .37])$ and claimants in the VA without MS condition $(M=.27, S D=.26,95 \%$ CI $[.20, .34])$ not differing in terms of the percentage of verifiable details they reported. However, the Veracity x Information Protocol Type interaction was significant $F(1,76)=7.39, \mathrm{MSE}=.374, p=0.008, \eta_{\mathrm{p}^{2}}=.09$. In the VA with MS condition, liars $(M=.07, S D=.10,95 \% \mathrm{CI}[-.03,0.18])$ reported a lower percentage of verifiable detail than truth tellers $(M=.52, S D=.26,95 \%$ CI $[.43, .62]), t(26,379)=7.431, p$ $<0.001, d=2.28$. Also in the VA without MS condition, liars $(M=.19, S D=.18,95 \% \mathrm{CI}$ $[.09, .28])$ reported a lower percentage of verifiable detail than truth tellers $(M=.36, S D=$ $.31,95 \%$ CI $[.26, .46), t(28.515)=-2.188, p=0.037, d=0.71$. Inspection of the effect sizes (Cohens $d$ ) shows the most pronounced between liars and truth tellers in the VA with MS 
condition (versus the VA without MS condition), which supports Hypothesis 3.

To test Hypothesis 3, we ran two discriminant analyses to distinguish between (i) claimants exposed to the VA with MS and (ii) claimants exposed to the VA without MS. In both case, the objective group belonging (truthful versus deceptive) was the classifying variable, with the percentage of verifiable detail entered as the predicator. As Table 1 shows, for the percentage of verifiable detail, a significant discriminant function emerged for distinguishing between truth tellers and liars exposed to the VA with MS, $\chi^{2}(1)=31.99$, Wilks' $\lambda=0.43, p<0.001$ (canonical correlation was .76). The function correctly classified $81.0 \%$ of the truth tellers and $100.0 \%$ of the liars resulting in an overall total accuracy rate of $90.0 \%$ of the claimants. A significant discriminant function also emerged for distinguishing between truth tellers and liars in the VA- IP without the model statement condition, $\chi^{2}(1)=$ 4.66, Wilks' $\lambda=0.88, p=0.031$ (canonical correlation was .34). The function correctly classified $52.6 \%$ of the truth tellers and $76.2 \%$ of the liars resulting in an overall total accuracy rate of $65.0 \%$ of the claimants. Thus, superior classification of truth tellers and liars emerged in the VA with MS condition, supporting Hypothesis 3.

[Insert Table 2 about here]

To complement the series of discriminant analyses, we also conducted a series of Receiver Operator Characteristic (ROC) analysis for verifiable, unverifiable and the percentage of verifiable detail. For verifiable and percentage of verifiable detail the state variable (i.e. the particular dichotomous outcome a positive test - i.e. a higher score indicates) was truth, and for unverifiable detail the state verifiable was lie. Unlike discriminate analysis, the Area Under the Curve (AUC) of a ROC curve (with 1- specificity, i.e. false positive rate, plotted on the $\mathrm{x}$-axis and sensitivity, i.e. true positive rate plotted on the y-axis) provides a measure of the diagnosticity of the criterion as a whole. As Figures 1a$3 \mathrm{~b}$ show, the area under the curve (AUC) obtained for each cue was greater in the VA with 
MS condition (.836 for verifiable, .962 unverifiable and .911 for the percentage of verifiable detail) than the AUC's obtained for each cue in the VA without MS condition (.569 for verifiable, .904 for unverifiable and .648 for the percentage of verifiable detail). This indicates superior diagnosticity for all examined cue in the VA with MS (versus VA without MS) condition.

[Insert Figures 1a-3b around here]

To establish the optimal cut-off point - the threshold value that maximises sensitivity and specificity - for each significant ROC analysis (Figures 1b, 2a, 2b and 3b) we computed the Youden index (Youden, 1950). This yielded an optimal cut-off point for verifiable details in the VA with MS condition of 29.0. For unverifiable details, the optimal cut-off point in the VA without MS condition was 30.5, whereas in VA with MS condition the optimal cut-off point for unverifiable details was 56.0. Finally, for the Percentage of verifiable detail in the VA with MS condition the optimal cut-off point was .305.

\section{Discussion}

The current study demonstrated that the addition of a model statement enhanced the ability of the VA to distinguish between fabricated and genuine statements within an insurance claims setting. Previous research has found discrimination between liars and truth tellers via popular content analysis (e.g. CBCA, RM, SCAN) to not be improved via interviewing with a model statement (Leal et al., 2016, experiment 2; Bogaard et al., 2014). The current findings break with this pattern. Indeed, consistent with predictions drawn from literature on the information management dilemma confronting liars and truth teller's forthcoming verbal strategies, the VA procedure supplemented with a model statement elicited from truthful claimants more verifiable detail compared to the VA procedure without a model statement. In contrast, providing or withholding the model statement had no effect upon the number of unverifiable details reported by truth tellers. As such, the VA with MS 
(versus VA without MS) elected only additional verifiable detail from truth tellers, rather than eliciting more information, including unverifiable details, in general. In contrast, providing or withholding the model statement had no effect upon the number of verifiable details reported by liars, but the VA with MS elicited more unverifiable detail from lying claimants compared to the VA without MS. This is easy to explain. If liars are simultaneously motivated to (i) provide detailed longer statements because of the model statement but (ii) avoid disclosing verifiable details, the only solution available is to disclose additional uncheckable details. It is worth speculating as to why unverifiable details emerged as such a distinctive factor across both experimental conditions. Plausibly, the IP component may make providing detail that is unverifiable especially attractive for liars (i.e. the strategically pragmatic path of least resistance during the interview), whereas providing unverifiable detail remains unattractive for truth tellers who know they are expected, and who are able, to provide checkable information (also see Vrij et al. 2016; c.f. Nahari et al., 2014a). Speculatively, the IP component may thus enhance the general utility of unverifiable details by reinforcing liars preferred strategies.

Given the reported pattern of findings, it is unsurprising that the percentage of verifiable detail per statement (verifiable/total detail) emerged as such a powerful cue to veracity in the VA with MS condition compared to the VA without MS condition, as the accuracy rate using the percentage of verifiable details as the cue to deceit raised from $65.0 \%$ in the VA without MS condition to $90.0 \%$ in the VA with MS condition. Given the well documented difficulty of lie detection in insurance claims settings (Harvey et al., 2016; Leal et al., 2015; Nahari, Leal et al., 2014; Vrij et al., 2016), this $90 \%$ accuracy rate is promising. Future research should isolate the cause of this enhancement. Theoretically, one possibility is that the enhancement is driven by the coupling of the model statement with the IP. If true, it is not the verifiable content of the model statement alone that responsible for the 
superior classificatory performance in the VA with MS condition, but the combination of MS and VA. Another possibility is that a model statement, by itself, motivates interviewees to disclose more detail generally (i.e. to report longer statements), and truth tellers to disclose more verifiable details specifically. That is, the length and the content of the model statement (i.e. verifiable details) attenuate the verbal behaviour of liars and truth tellers. If true, then just providing interviewees with a model statement comprised of checkable detail without the standard IP instruction may be effective. If effective, such a protocol would have the applied benefit of eliciting checkable detail from truth tellers whilst not disclosing explicitly to the interviewee what the interviewer wants to know. This warrants future research.

Given previous research (e.g. Harvey et al., 2016; Vrij et al., 2016), it is puzzling that liars and truth tellers in the VA without MS condition did not differ significantly in terms of verifiable detail. However, the setting a credibility assessment technique is applied to is critical, and generalising from one setting to another is problematic (Vrij et al., 2012). With respect to the current study, its noteworthy that individuals tend to lie more often while conversing over the phone, comparing to conversing in person (face-to-face) (DePaulo, Kashy, Kirkendol, Wyer, \& Epstein, 1996; Hancock, Thom-Santelli, \& Ritchie, 2004), and when writing an email comparing to writing a letter via pen and paper (Naquin, Kurtzberg and Belkin, 2010). Previous VA research has employed a face-to-face or phone interviewing setting, and the current study explored a computer mediated communication (CMC) setting. Plausibly, the different forensic settings examined in the current study could be responsible for the small-medium effect size observed for verifiable details in the VA without MS condition Future research should examine this possibility.

It could be argued that it is somewhat counter-intuitive that liars did not employ more embedding (basing deception upon genuine events; Leins, Fisher \& Ross, 2013) to enable disclosure of additional verifiable details, especially in the VA with MS condition. However, 
previous research has shown that providing verifiable details is, for liars, a difficult task (Nahari et al., 2014a, 2014b) even when an opportunity for embedding is presented (Nahari \& Vrij, 2015). For example, when deceivers were presented with a scenario that specifically allowed them to embed their lies and provide verifiable details, $60 \%$ did not draw upon the legitimate opportunity to disclose checkable details (Nahari \& Vrij, 2015). Similarly, liars in the current study reported providing statements containing a significant proportion $(29.75 \%$ of their overall statement) of embedded (truthful) detail, yet failed to match truth tellers in terms of the number of reported verifiable details. This finding is also observed in other studies (Harvey et al., 2016; Nahari et al., 2014). One potential explanation is that verifiable detail drawn from embedded lies remains an adverse strategy that liars are highly motivated to avoid (Granhag \& Hartwig, 2008; Hartwig et al., 2010; Nahari et al., 2014a, 2014b; Nahari \& Vrij, 2015). Such verifiable detail potentially risks exposing a liar's deception if investigated. For example, traceable friends must support the liar's statement (i.e. being at a specific place at a given time) that requires cooperation and coordination (Nahari \& Vrij, 2014; Nahari \& Vrij, 2015). This is a dangerous strategy and makes the deception more complex (whereas liars prefer to keep their stories simple; Hartwig et al., 2010) and includes the risk for friends to introduce between-statement inconsistencies (i.e., the friend contradicting the statement of the liar; Vrij et al., 2010). Such difficulties are also observed in Nahari and Vrij (2015), alongside being able finding a witness, being able to trust a witness, issues with timing, creating of evidence and prediction and control. Furthermore, liars may be reluctant to draw others into their deception because it makes clear to their friends that they are deceiving the insurance company for personal gain. They may anticipate social sanctions from their friends. As such, resorting to the disclosure of additional unverifiable details in the VA with MS condition appeared to be their preferred strategy.

It could be argued that liars in organised criminal networks may be willing to prepare 
and execute lies for each other (e.g. falsely claiming to have witnessed the fabricated event). In such cases, investigators could ask the interviewee(s) to demonstrate they were together when they claimed to have been together. Research has shown that liars, struggle to match the quantity of checkable information disclosed by truth tellers showing that they have been together, which allowed the VA to distinguish $96 \%$ of deceptive pairs and $80 \%$ of the truthful pairs correctly (Nahari \& Vrij, 2014). Even when liars are provided with an opportunity to embed their lies, providing checkable detail that match the quantity of those of a truth teller, is still a difficult task for liars (Nahari \& Vrij, 2015).

In conclusion, the current findings extend the previous literature showing that imposing proactive manipulations during an interview can magnify the verbal differences between liars and truth tellers when evaluated with the VA. Furthermore, the positive effects obtained by priming are theoretically grounded and based upon the different verbal strategies employed by liars and truth tellers. Whereas truth tellers could report verifiable details to match the model statement's level of verifiable detail, the only strategy consistent with the information management dilemma available to liars is to report more unverifiable detail. Therefore, priming interviewees to report verifiable details appears to be a useful addition, and potential refinement, to the full VA procedure. 


\section{References}

Association of British Insurers (2009). Research brief general insurance claims fraud. Retrieved from http://www.abi.org.uk/Publications/55680.pdf

Bell, B. E., \& Loftus, E. F. (1988). Degree of detail of eyewitness testimony and mock juror judgments. Journal of Applied Social Psychology, 18, 1171-1192. doi:10.1037/00223514.56.5.669

Bogaard, G., Meijer, E. H., \& Vrij, A. (2014). Using an example statement increases information but does not increase accuracy of CBCA, RM, and SCAN. Journal of Investigative Psychology and Offender Profiling, 11(2), 151-163. doi:10.1002/jip.1409

Cialdini, C. B. (1994). Influence: The psychology of persuasion (revised edition). Quill, New York.

Daft, R. L., \& Lengel, R. H. (1986). Organizational information requirements, media richness and structural design. Management science, 32(5), 554-571.

DePaulo, B. M., Kashy, D. A., Kirkendol, S. E., Wyer, M. M., \& Epstein, J. A. (1996). Lying in everyday life. Journal of personality and social psychology, 70(5), 979.

DePaulo, B. M., Lindsay, J. J., Malone, B. E., Muhlenbruck, L., Charlton, K., \& Cooper, H. (2003). Cues to deception. Psychological Bulletin, 129(1), 74. doi:10.1037/00332909.129.1.74

Ekman, P. (1985). Telling lies: Clues to deceit in the marketplace, marriage, and politics. 
Festinger, L. (1954). A theory of social comparison processes. Human relations, 7, 117-140. doi: $10.1177 / 001872675400700202$

Gilovich, T., Savitsky, K., \& Medvec, V. H. (1998). The illusion of transparency: biased assessments of others' ability to read one's emotional states. Journal of personality and social psychology, 75(2), 332. doi:10.1037/0022-3514.75.2.332

Granhag, P. A., \& Hartwig, M. (2008). A new theoretical perspective on deception detection: On the psychology of instrumental mind-reading. Psychology, Crime \& Law, 14, 189-200. doi:10.1080/ 10683160701645181

Hancock, J. T., Thom-Santelli, J., \& Ritchie, T. (2004, April). Deception and design: The impact of communication technology on lying behavior. In Proceedings of the SIGCHI conference on Human factors in computing systems (pp. 129-134). ACM.

Hartwig, M., \& Bond Jr, C. F. (2011). Why do lie-catchers fail? A lens model meta-analysis of human lie judgments. Psychological bulletin, 137(4), 643. doi:10.1037/a0023589

Hartwig, M., Anders Granhag, P., \& Strömwall, L. A. (2007). Guilty and innocent suspects' strategies during police interrogations. Psychology, Crime \& Law, 13(2), 213-227. doi:10.1080/10683160600750264

Hartwig, M., Granhag, P. A., Stromwall, L. A., \& Doering, N. (2010). Impression and information management: On the strategic self-regulation of innocent and guilty suspects. Open Criminology Journal, 3, 10-16. doi:10.2174/1874917801003020010

Hartwig, M., Granhag, P. A., Strömwall, L. A., \& Vrij, A. (2005). Detecting deception via strategic disclosure of evidence. Law and human behavior, 29(4), 469. doi:10.1007/s10979-005-5521-x 
Harvey, A. C., Vrij, A., Nahari, G., \& Ludwig, K. (2016). Applying the Verifiability Approach to insurance claims settings: Exploring the effect of the information protocol. Legal and Criminological Psychology. doi: 10.1111/lcrp.12092

Johnson, M. K., \& Raye, C. L. (1981). Reality monitoring. Psychological review, 88(1), 67. doi:10.1037/0033-295X.88.1.67

Johnson, M. K., Foley, M. A., Suengas, A. G., \& Raye, C. L. (1988). Phenomenal characteristics of memories for perceived and imagined autobiographical events. Journal of Experimental Psychology: General,117(4), 371. doi: $10.1037 / 0096-3445.117 .4 .371$

Leal, S., Vrij, A., Warmelink, L., Vernham, Z., \& Fisher, R. (2015). You cannot hide your telephone lies: Providing a model statement as an aid to detect deception in insurance telephone calls. Legal and Criminological Psychology, 20, 129-146. doi:10.1111/lcrp.12017

Leins, D. A., Fisher, P. F., \& Ross, S. J. (2013). Exploring liars'strategies for creating deceptive reports. Legal and Criminological Psychology, 18, 141-151. doi:10.1111/j.2044-8333.2011. 02041.x

Levine, T. R. (2014). Active deception detection. Policy Insights from the Behavioral and Brain Sciences, 1, 122-128. doi:10.1177/2372732214548863

Masip, J., \& Herrero, C. (2013). 'What would you say if you were guilty?' Suspects' strategies during a hypothetical behavior analysis interview concerning a serious crime. Applied Cognitive Psychology, 27, 60-70. doi:10.1002/acp.2872

Nahari, G. (2016). When the long road is the shortcut: A comparison between two coding 
methods for content-based lie detection tools. Psychology, Crime and Law, 22, 10001014. Nahari, G., \& Vrij, A. (2014). Can I borrow your alibi? The applicability of the verifiability approach to the case of an alibi witness. Journal of Applied Research in Memory and Cognition, 3, 89-94. doi:10.1016/j.jarmac.2014.04.005

Nahari, G., \& Vrij, A. (2015). Can someone fabricate verifiable details when planning in advance? It all depends on the crime scenario. Psychology, Crime \& Law, 21, 987999. doi:10.1080/ 1068316X.2015.1077248

Nahari, G., Leal, S., Vrij, A., Warmelink, L., \& Vernham, Z. (2014). Did somebody see it? Applying the verifiability approach to insurance claims interviews. Journal of Investigative Psychology and Offender Profiling, 11, 237-243. doi:10.1002/jip.1417

Nahari, G., Vrij, A., \& Fisher, R. P. (2014a). Exploiting liars' verbal strategies by examining the verifiability of details. Legal and Criminological Psychology, 19, 227-239. doi:10.1111/j.2044- 8333.2012.02069.x

Nahari, G., Vrij, A., \& Fisher, R. P. (2014b). The verifiability approach: Countermeasures facilitate its ability to discriminate between truths and lies. Applied Cognitive Psychology, 28, 122-128. doi:10.1002/acp.2974

Shaw, D. J., Vrij, A., Leal, S., Mann, S., Hillman, J., Granhag, P. A., \& Fisher, R. P. (2015). Mimicry and investigative interviewing: Using deliberate mimicry to elicit information and cues to deceit. Journal of Investigative Psychology and Offender Profiling, 12(3), 217-230. doi: 10.1002/jip.1438

Strömwall, L. A., Hartwig, M., \& Granhag, P. A. (2006). To act truthfully: Nonverbal behaviour and strategies during a police interrogation. Psychology, Crime \& Law, 12(2), 207-219. doi: 10.1080/10683160512331331328 
Vrij, A., Mann, S., Kristen, S., \& Fisher, R. P. (2007). Cues to deception and ability to detect lies as a function of police interview styles. Law and human behavior, 31(5), 499-518. doi: 10.1007/s10979-006-9066-4

Vrij, A. (2008). Detecting lies and deceit: Pitfalls and opportunities. Chichester, UK: John Wiley \& Sons.

Vrij, A. (2015). Verbal Lie Detection tools: Statement validity analysis, reality monitoring and scientific content analysis. In P. A. Granhag, A. Vrij, \& B. Verschuere. (Eds.) Detecting Deception Current Challenges and Cognitive Approaches. Chichester: John Wiley \& Sons.

Vrij, A., \& Granhag, P. A. (2012). Eliciting cues to deception and truth: What matters are the questions asked. Journal of Applied Research in Memory and Cognition, 1, 110-117. doi:10.1016/j.jarmac.2012.02.004

Vrij, A., \& Granhag, P. A. (2014). Eliciting information and detecting lies in intelligence interviewing: An overview of recent research. Applied Cognitive Psychology, 28, 936-944. doi:10.1002/ acp.3071

Vrij, A., Mann, S., Leal, S., \& Granhag, P. (2010). Getting into the minds of pairs of liars and truth tellers: An examination of their strategies. Open Criminology Journal, 3(1), 1722. doi: $10.2174 / 1874917801003010017$

Vrij, A., Nahari, G., Isitt, R., \& Leal, S. (2016). Using the verifiability lie detection approach in an insurance claim setting. Journal of Investigative Psychology and Offender Profiling. doi: 10.1002/jip.1458

Youden, W. J. (1950), Index for rating diagnostic tests. Cancer, 3: 32-35. doi:10.1002/10970142(1950)3:1<32::AID-CNCR2820030106>3.0.CO;2-3 


\section{Table 1}

Breakdown of the IP components and instructions

IP Component $\quad$ IP Instruction

(I) Reporting

We know from research that liars prefer to avoid providing details that can be verified whereas truth tellers prefer to provide details that can be verified.

(II) Analysis

Therefore the analyst will check carefully to what extent the details you provide can be verified.

(III) Definition

Verifiable details are i) activities carried out with identifiable or named persons who the interviewer can consult, ii) activities that have been witnessed by identifiable or named persons who the interviewer can consult, iii) activities that the interviewee believes may have been captured on CCTV and iv) activities that may have been recorded and documented, such as using debit cards, mobile phones, or computers.

(IV) Falsifiability

The analyst may check the occurrence of some or all of the details provided in the statements after the interview.

Note: Adapted from Nahari, G., Vrij, A., \& Fisher, R. P. (2014b). The verifiability approach: Countermeasures facilitate its ability to discriminate between truths and lies. Applied Cognitive Psychology, 28, 122-128. doi:10.1002/acp.2974 
Table 2

Hit Rates for the percentage of verifiable detail per statement (verifiable/ total detail) as a function of $V A$ procedure.

$\begin{array}{ll}\text { VA without } & \text { VA with } \\ \text { MS } & \text { MS }\end{array}$

$\begin{array}{llllll}\text { Truthful } & \text { Deceptive } & \text { Total } & \text { Truthful } & \text { Deceptive } & \text { Total } \\ (\%) & (\%) & (\%) & (\%) & (\%) & (\%)\end{array}$

Verifiable/ total

detail

52.6

76.2

65.0

81.0

100.0

90.0

Accuracy rate

Note. Accuracy rates from significant discriminate functions appear in bold. 
Figure captions

Figure 1a: ROC curve (with AUC) for verifiable detail in the VA with no MS condition

Figure 1b: ROC curve (with AUC) for verifiable detail in the VA with MS condition

Figure 2a: ROC curve (with AUC) for unverifiable detail in the VA with no MS condition

Figure 2b: ROC curve (with AUC) for unverifiable detail in the VA with MS condition

Figure 3a: ROC curve (with AUC) for the percentage of verifiable detail in the VA with no MS condition

Figure 3b: ROC curve (with AUC) for the percentage of verifiable detail in the VA with MS condition 


\section{STATEMENT COMPONENT}

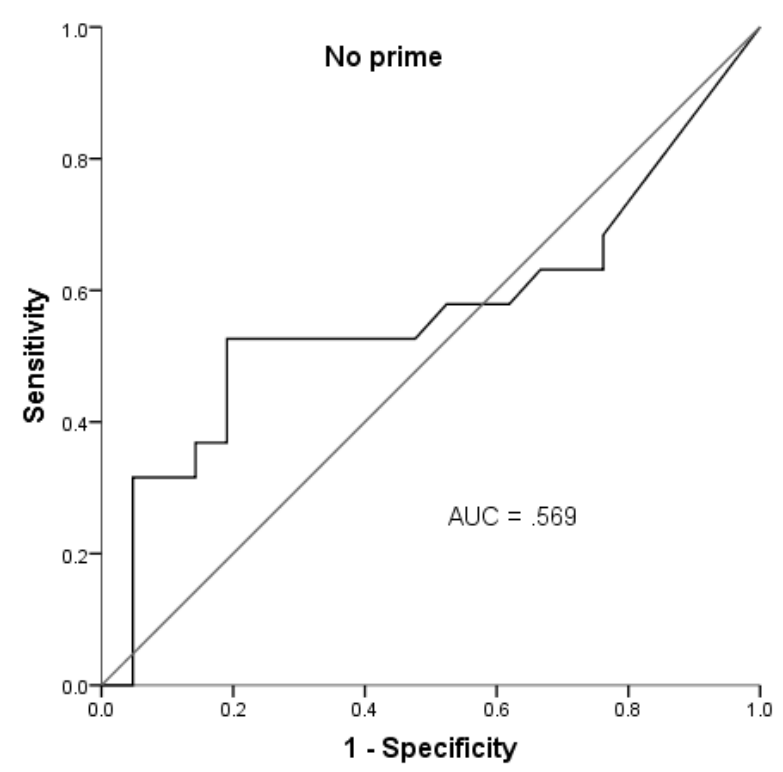

Figure 1a

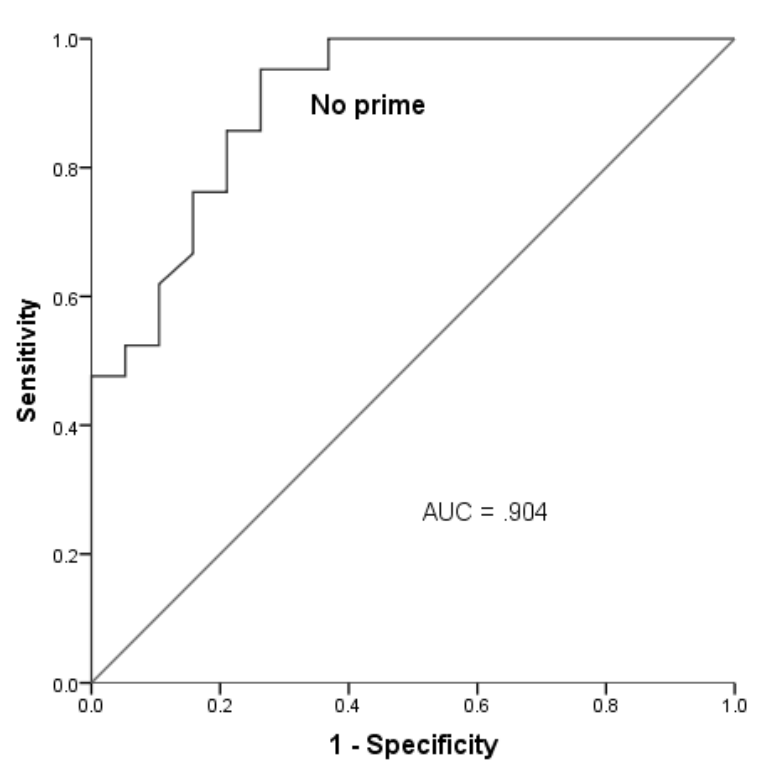

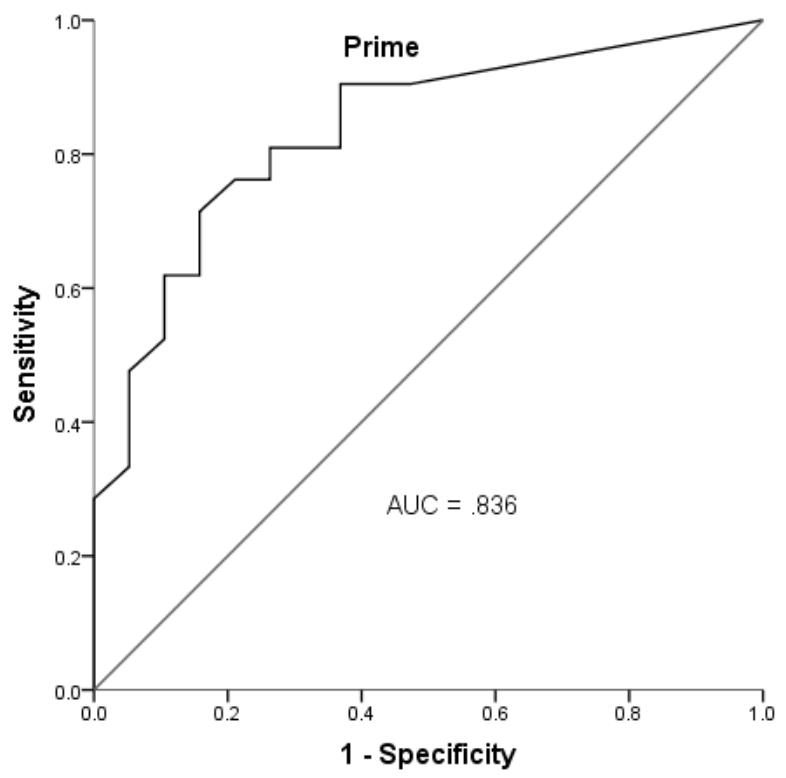

Figure 1b

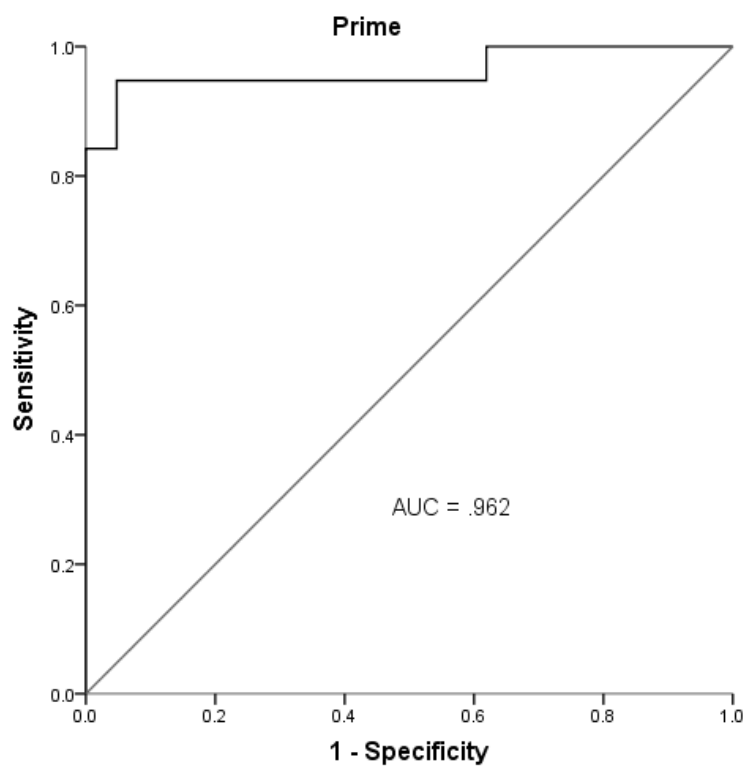

Figure 2a

Figure $2 b$ 


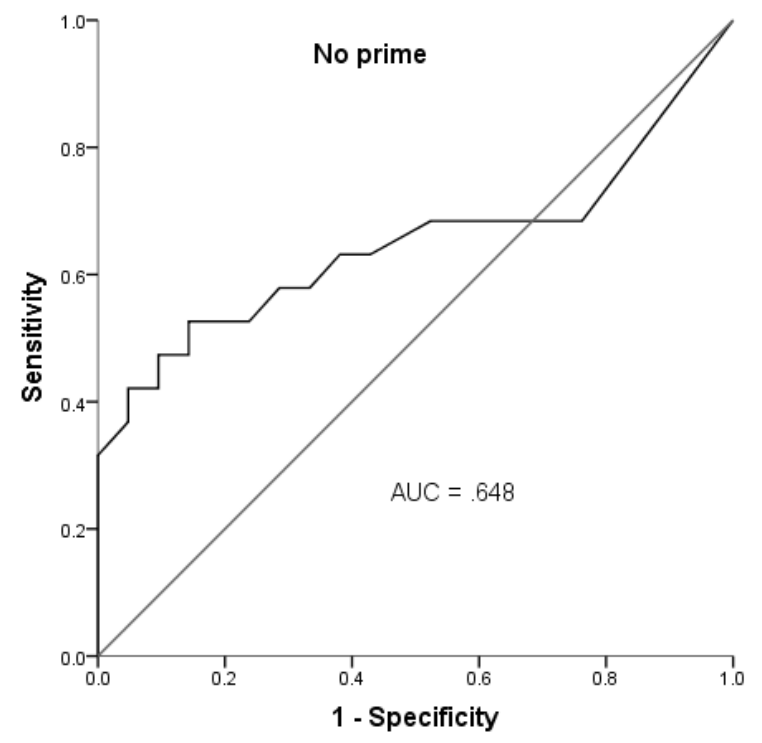

Figure 3a

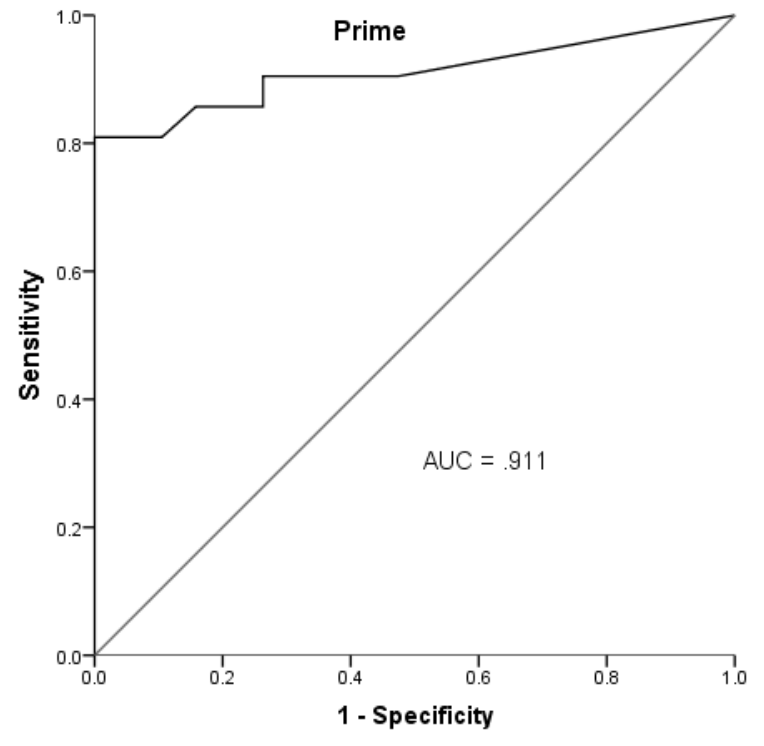

Figure $3 b$ 